\title{
Erratum to rhinovirus is associated with severe adult community-acquired pneumonia in China
}

\author{
Keqiang Wang ${ }^{1 \#}$, Wen $\mathrm{Xi}^{1 \#}$, Donghong Yang ${ }^{1 \#}$, Yali Zheng ${ }^{1 \#}$, Yaqiong Zhang ${ }^{2}$, Yusheng Chen ${ }^{3}$, \\ Chunliang Yan ${ }^{4}$, Guizhen Tian ${ }^{5}$, Shuchang $\mathrm{An}^{6}$, Xiangxin $\mathrm{Li}^{7}$, Fucheng An ${ }^{8}$, Juan $\mathrm{Du}^{9}, \mathrm{Ke} \mathrm{Hu}^{10}$, Jie $\mathrm{Cao}^{11}$, \\ Lili Ren ${ }^{12}$, Fang Huang ${ }^{13}$, Zhancheng Gao ${ }^{1}$ \\ ${ }^{1}$ Department of Respiratory and Critical Care Medicine, Peking University People's Hospital, Beijing 100044, China; ${ }^{2}$ Department of Respiratory \\ and Critical Care Medicine, Peking University International Hospital, Beijing 102206, China; ${ }^{3}$ Department of Respiratory Medicine, Fujian \\ Provincial Hospital, Fuzhou 350001, China; ${ }^{4}$ Department of Respiratory Medicine, Beijing Aerospace General Hospital, Beijing 100076, China; \\ ${ }^{5}$ Department of Respiratory Medicine, 263 Hospital of the Chinese People's Liberation Army, Beijing 101149, China; ${ }^{6}$ Department of Respiratory \\ Medicine, The First Hospital of Tsinghua University, Beijing 100016, China; ${ }^{7}$ Department of Respiratory Medicine, Beijing Changping Hospital, \\ Beijing 102200, China; ${ }^{8}$ Department of Respiratory Medicine, Beijing Mentougou District Hospital, Beijing 102300, China; ${ }^{9}$ Department of \\ Respiratory Medicine, The Affiliated Hospital of Guiyang Medical College, Guiyang 550004, China; ${ }^{10}$ Department of Respiratory Medicine, Renmin \\ Hospital of Wuhan University, Wuhan 430060, China; ${ }^{11}$ Department of Respiratory Medicine, Tianjin Medical University General Hospital, Tianjin \\ 300052, China; ${ }^{12} \mathrm{MOH}$ Key Laboratory of Systems Biology of Pathogens and Christophe Mérieux Laboratory, IPB, CAMS-Fondation Mérieux, \\ Institute of Pathogen Biology (IPB), Chinese Academy of Medical Sciences (CAMS) \& Peking Union Medical College, Beijing 100730, China; \\ ${ }^{13}$ Beijing Center for Disease Prevention and Control, Beijing 100013, China \\ \#These authors contributed equally to this work. \\ Correspondence to: Zhancheng Gao, MD, PhD. Department of Respiratory and Critical Care Medicine, Peking University People's Hospital, Beijing \\ 100044, China. Email: zcgao@bjmu.edu.cn.
}

doi: $10.21037 /$ jtd.2018.11.11

View this article at: http://dx.doi.org/10.21037/jtd.2018.11.11

Erratum to: J Thorac Dis 2017;9:4502-11

Rhinovirus is associated with severe adult community-acquired pneumonia in China

In the article that appeared on Page 4502-4511, Vol 9, No 11 (November 2017) Issue of the fournal of Thoracic Disease (fTD) (1), Dr. Lili Ren's affiliation was incorrect. The correct affiliation should be: MOH Key Laboratory of Systems Biology of Pathogens and Christophe Mérieux Laboratory, IPB, CAMS-Fondation Mérieux, Institute of Pathogen Biology (IPB), Chinese Academy of Medical Sciences (CAMS) \& Peking Union Medical College, Beijing 100730, China.

The authors regret the error.

\section{References}

1. Wang $\mathrm{K}$, Xi W, Yang D, et al. Rhinovirus is associated with severe adult community-acquired pneumonia in China. J Thorac Dis 2017;9:4502-11.

Cite this article as: Wang $\mathrm{K}$, $\mathrm{Xi}$ W, Yang D, Zheng Y, Zhang Y, Chen Y, Yan C, Tian G, An S, Li X, An F, Du J, Hu K, Cao J, Ren L, Huang F, Gao Z. Erratum to rhinovirus is associated with severe adult community-acquired pneumonia in China. J

Thorac Dis 2018;10(12):E861. doi: 10.21037/jtd.2018.11.11 